ISSN 1112-9867

Available online at

http://www.jfas.info

\title{
QUALITY OF IMPORTED HONEYS MARKETED IN ALGERIA
}

\author{
L. Haderbache*1, A. Mohammedi ${ }^{2}$ \\ ${ }^{1}$ Laboratoire de recherche en technologies alimentaires, Université Boumerdes 35000 \\ ${ }^{2}$ Laboratoire de valorisation et conservation des ressources biologiques, Université
}

Boumerdes 35000

Received: 25 November 2014 / Accepted: 05 January 2015 / Published online: 15 January 2015

\begin{abstract}
Honey is mostly used for therapeutic purposes, that's why its quality must be closely monitored. This study focuses on the controle of 40 imported honeys coming from 13 countries (2004 2011). We revealed that they do not always have the required quality to be consumed as such. In addition to the labeling and organoleptic problems, often they contains too much moisture ( $\geq 18 \%$ ); $70 \%$ of these samples had an average HMF of $78 \mathrm{mg} / \mathrm{kg}$ and a $\mathrm{pH}$ less than 3.5, indicating an advanced aging and inapropriate thermal past. Pollen analysis and electrical conductivity was used to confirm presumed origins, and proline rate reveals some inverted sugar fraud. We aim by this work to catch consumers and local authorities attention in order to strengthen honey control. It is about everyone's health, and it concerns the prosperity of the national beekeeping which faces unfair competition.
\end{abstract}

Keywords: fraud; physico-chemical; pollinic; consumer protection; control.

\section{INTRODUCTION}

Le miel est un aliment produit par l'abeille mellifère et sensé être consommé sans aucune transformation, il est considéré comme supplément alimentaire pour sa richesse en composés utiles tel que le $\alpha$ tocophérol, l'acide ascorbique, les flavonoïdes et les composés phénoliques, lui procurant son pouvoir antioxydant et antibactérien [1] mais c'est aussi une source pure d'énergie rapide vu qu'il est constitué à $80 \%$ de sucres.

Author Correspondence, e-mail: latifahaderbache@yahoo.fr

Tel. : +213559332209; Fax : +21324911116

ICID: 1134460 
C'est une matrice complexe influencée par l'origine géographique, les conditions pédoclimatiques, les conditions de manutention et de stockage mais surtout par la source florale butinée, qui lui confère sa touche aromatique tant appréciée par le consommateur [2].

On voit, aujourd'hui, sur nos étalages tout type de produits alimentaires d'importation, originaire de pratiquement tous les pays du monde. Cet afflux de denrées se fait anarchiquement devant des systèmes de contrôle insuffisant pour assurer la protection du consommateur. Ceci sans prendre en considération les répercussions sur la petite et moyenne entreprise algérienne, qui se trouvent concurrencées par des producteurs de l'étranger, offrant une plus large gamme de produits avec une meilleure présentation et des prix plus intéressants.

L'exemple du secteur apicole est l'un des plus parlants, alors que la profession, avec l'aide de l'état, s'efforce, depuis une quinzaine d'années, à développer cette branche de d'activité, le marché de l'importation s'ouvre et décourage la production. L'importation du miel est devenue une pratique commerciale de plus en plus courante, selon les chiffres donnés par les Douanes algérienne, elle est passée de 18 tonnes en 1999 à 800 tonnes en 2010 [3], ceci sans comptabiliser les quantités circulant dans le circuit informel et entrant dans le pays d'une manière illicite.

Avec des niveaux de production de l'ordre de 1500 tonnes en 1990 et 4100 tonnes en 2010 [3], la production nationale de miel est loin de satisfaire la demande croissante du consommateur qui comble ce besoin par des produits d'importation. Sans pour autant être de très grande qualité, ils offrent l'avantage d'être disponibles à longueur d'année et d'être bon marché, mais sont-ils conformes aux critères du Codex Alimentarius ?

Le but de ce travail est, justement, de répondre aux interrogations des un et des autres concernant ces denrées importées et de donner au consommateur une image de ce qu'il est entrain de consommer.

\section{MATERIELS ET METHODES}

\subsection{Collecte des miels importés}

L'étude a porté sur 40 échantillons de miels importés collectés entre 2004 et 2011, sur les étalages des magasins et des supermarchés c'est-à-dire déjà mis à la disposition du consommateur, ou rapportés par des particuliers, cherchant à importer du miel ou l'ayant déjà introduit sur le territoire national.

Les échantillons ont subit une batterie d'analyses de qualité et de contrôle de l'appellation après avoir été décrit grâce aux informations mentionnées sur l'étiquetage s’ils existent et s'ils sont lisibles (Nom commercial, Nom du producteur, pays d'origine, poids, date de production, date 
de péremption, prix par pot) ; d'autres descripteurs sont noté tel que la couleur, l'état de cristallisation et les défauts apparents.

L'échantillonnage a pris en considération les différents types d'emballages et les différentes contenances, et pour des raisons pratiques, les échantillons ont été codés et conservés à $4^{\circ} \mathrm{C}$ jusqu'au moment de l'analyse qui n'a pas dépassé un mois.

\subsection{Matériel et Méthodes}

Les méthodes d'analyse adoptées sont les méthodes harmonisées d'analyse des miels de l'International Honey Commission [4] équivalentes aux méthodes AOAC. L'humidité, le pH, l'acidité libre (AL), la conductivité électrique (CE), le taux d'hydroxyméthylfurfural (HMF), la proline et la couleur Pfund ont été mesurés.

L'origine botanique mentionnée sur l'emballage est vérifiée par l'analyse pollinique [5], les pollens sont identifiés grâce aux photos de pollens de référence du text book of melissopalynology [6].

\section{RESULTATS ET DISCUSSION}

Le tableau 1 décrit ces échantillons et les photographies fig. 1 et 2 montrent les différents matériaux d'emballage et les différents volumes et présentations proposés au consommateur.

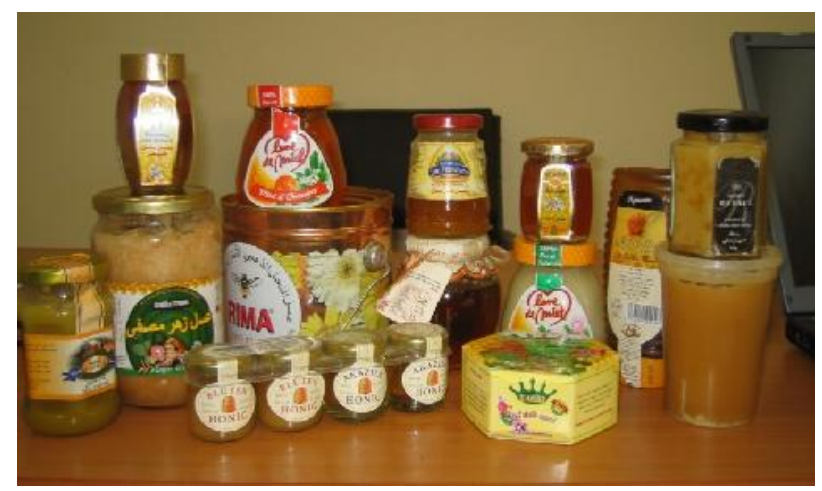

Fig.1. Présentation des échantillons de miel importés.

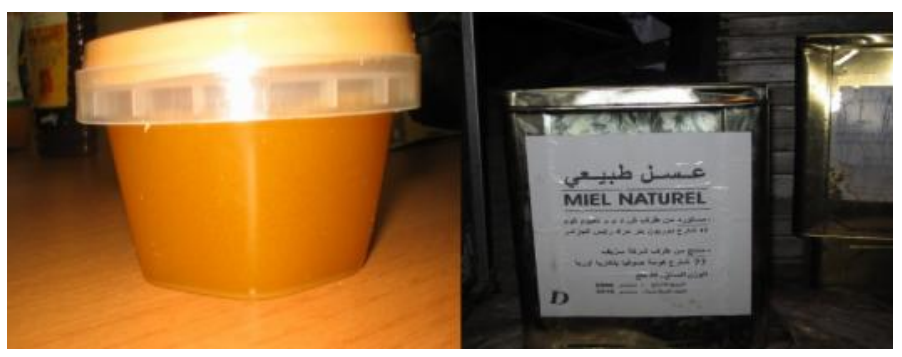

Fig.2. Echantillon (gauche) emballé dans un pot en plastique, et un autre (droite) dans un fut métallique. 
Tableau 1 : Description générale des échantillons de miels importés collectés pour l'étude

\begin{tabular}{|c|c|c|c|c|c|}
\hline $\mathbf{N}^{\circ}$ & Nom commercial & $\begin{array}{l}\text { Origine florale } \\
\text { supposée }\end{array}$ & Pays d'origine & $\begin{array}{r}\text { Poids } \\
(\mathrm{g})\end{array}$ & $\begin{array}{c}\text { Date de } \\
\text { production }\end{array}$ \\
\hline 1 & Granja San Francisco & TF & Espagne & 250 & 2006 \\
\hline 2 & Jena miel & $\mathrm{TF}$ & Espagne & 350 & 2006 \\
\hline 3 & Martelet & Honey royal & Grande Bretagne & 250 & 2006 \\
\hline 4 & Langese & $\mathrm{TF}$ & Allemagne & 125 & 2004 \\
\hline 5 & Imker anstese & Acacia & Allemagne & 50 & 2006 \\
\hline 6 & Imker antese & Bluten & Allemagne & 50 & 2006 \\
\hline 7 & Alshiffa & $\mathrm{TF}$ & Arabie Saoudite & 125 & 2006 \\
\hline 8 & L'abeille & TF & Bulgarie & 250 & 2006 \\
\hline 9 & Metin & TF & Turquie & 450 & 2007 \\
\hline 10 & Al baraka & TF & Importé & 140 & 2006 \\
\hline 11 & Flower honey-shiffa home & $\mathrm{TF}$ & Turquie & 1000 & 2004 \\
\hline 12 & L'abeille & TF & Bulgarie & 25000 & 2006 \\
\hline 13 & Lune de miel & Miel de Trèfle & France & 375 & 2006 \\
\hline 14 & Lune de miel & Miel d'Oranger & France & 375 & 2006 \\
\hline 15 & Rima & TF & Australie & 1500 & 2005 \\
\hline 16 & - & Miel de foret & Mali & 40000 & 2006 \\
\hline 17 & San francisco & $\mathrm{TF}$ & Espagne & 125 & 2010 \\
\hline 18 & San francisco & $\mathrm{TF}$ & Espagne & 125 & 2010 \\
\hline 19 & - & TF & Mali & 1000 & 2010 \\
\hline 20 & - & $\mathrm{TF}$ & Cote d'ivoire & 1000 & 2011 \\
\hline 21 & - & $\mathrm{TF}$ & Cote d'ivoire & 1000 & 2011 \\
\hline 22 & - & $\mathrm{TF}$ & Cote d'ivoire & 1000 & 2011 \\
\hline 23 & - & $\mathrm{TF}$ & Cote d'ivoire & 1000 & 2011 \\
\hline 24 & - & TF & Cote d'ivoire & 1000 & 2011 \\
\hline 25 & - & $\mathrm{TF}$ & Inde & 1000 & 2011 \\
\hline 26 & Al shiffa & Toute Fleurs & Arabie saoudite & 500 & 2010 \\
\hline 27 & San francisco & Toute Fleurs & Espagne & 250 & 2008 \\
\hline 28 & San francisco & $\mathrm{TF}$ & Espagne & 500 & 2011 \\
\hline 29 & - & Foret & Mali & 25000 & 2011 \\
\hline 30 & San francisco & $\mathrm{TF}$ & Espagne & 250 & 2011 \\
\hline 31 & - & Foret & Niger & 40000 & 2011 \\
\hline 32 & - & $\mathrm{TF}$ & Etranger & 1000 & 2011 \\
\hline 33 & - & $\mathrm{TF}$ & Etranger & 1000 & 2011 \\
\hline 34 & San francisco & Toute Fleur & Espagne & 250 & 2011 \\
\hline 35 & Al shiffa & Toute Fleur & Arabie saoudite & 125 & 2011 \\
\hline 36 & Langnese & Foret & Allemagne & 125 & 2010 \\
\hline 37 & Miel de nectar & colza & Allemagne & 50 & 2010 \\
\hline 38 & Miellat & Montagne & Allemagne & 50 & 2010 \\
\hline 39 & Miel nectar & Acacia & Espagne & 125 & 2010 \\
\hline 40 & Miel nectar & Jujubier & Kachmir & 1000 & 2010 \\
\hline
\end{tabular}

- Absence d'information ; TF : toute fleur ; 


\subsection{Conformité commerciale et légale des échantillons analysés}

Les miels collectés sont originaires de 13 pays, dont quatre de l'union européenne, trois d'Afrique, 5 du moyen orient, d'Asie de l'Ouest et d'Australie. Près de $48 \%$ des échantillons sont correctement étiquetés et portent mention de l'origine florale ou géographique, les autres présentent des défauts majeurs d'étiquetage (absence de la dénomination du produit, de la date de production, de la DLUO, du pays d'origine, du poids net). Ce manque d'informations ne permet pas une bonne traçabilité du produit ou pouvant créer une confusion chez le consommateur, nous donnons l'exemple de deux miels l'un d'Arabie saoudite dénommé «al shiffa » et un autre provenant de Turquie dénommé «shiffa » de shiffa home.

D'autres échantillons portent des mentions en langue étrangère (allemand ou turque) qui rendent difficile le déchiffrage des informations ce qui est contraire à la réglementation nationale concernant l'étiquetage des produits importés (Art 7. JORA N ${ }^{\circ} 58$ du 9 novembre 2013, décret $\left.N^{\circ} 13 / 378\right)$.

Les miels en question sont présentés sous divers emballages (verre, plastique ou fer blanc), avec des contenances allant du petit pot de $50 \mathrm{~g}$ au bidon de $40 \mathrm{~kg}$, mais dans la majeure partie des cas ils sont bien emballés dans du verre avec un étiquetage entreillant et un emballage pratique. Le prix ramené au kilogramme varie entre 333 et 630 DA (Turquie et Australie) et 2143 DA (Arabie saoudite) avec une moyenne autour de 1136,2 \pm 489,1 DA, ce qui présente une concurrence sure aux miels locaux vu que les prix proposés par nos apiculteurs dépassent le seuil de 2500 DA dans le meilleur des cas.

\subsection{Conformité organoleptique}

Le tableau 2 donne la description sensorielle de quelques uns de ces miels, $43 \%$ de ces échantillons présentent des défauts de cristallisation bien décrits par Gonnet [2] (figure 3). Ceux là sont dus soit à un traitement thermique inadéquat, soit à une mauvaise homogénéisation ou une fermentation. On retrouve des signes de mauvais traitement comme la séparation de phase issue d'une absorption d'humidité, les taches brunes dues à une contamination, les marbrures blanches et les cristallisations aléatoires indiquant des chocs thermiques (froids ou chauds), les débris de cires ou les bulles d'air provenant d'un mauvais écumage et un mauvais conditionnement, et enfin les odeurs de fermentation pour les miels trop humides et qui est l'accident de conservation le plus grave car il entraine une insalubrité du produit.

Du point de vu couleur et consistance, près de $60 \%$ de ces miels sont ambrés foncés et fluides, type de miel, justement, très recherché par le consommateur algérien d'après une enquête que nous avons mené [8]. 
Tableau 2: Description sensorielle et défauts d'apparence de quelques miels importés

\begin{tabular}{|c|c|c|c|c|c|}
\hline $\mathbf{N}^{\circ}$ & Couleur & Aspect & Contenant & Observations générales & Diagnostic \\
\hline 1 & foncé & $\begin{array}{l}\text { En phase de } \\
\text { cristallisation }\end{array}$ & verre & $\begin{array}{l}\text { Défaut de cristallisation } \\
\text { (séparation des phases). }\end{array}$ & $\begin{array}{l}\text { Prise d'humidité du à un } \\
\text { mauvais stockage ou à un } \\
\text { défaut d'embalage. }\end{array}$ \\
\hline 2 & foncé & $\begin{array}{l}\text { Liquide au } \\
\text { départ puis } \\
\text { cristallisé }\end{array}$ & plastique & Cristallisation homogène & - \\
\hline 3 & clair & Cristallisé & verre & $\begin{array}{l}\text { Présence de défaut de } \\
\text { cristallisation (des taches } \\
\text { brunes) Présence de bulle } \\
\text { d'air au fond du pot sous } \\
\text { forme d'une couche } \\
\text { continue horizontale. }\end{array}$ & $\begin{array}{l}\text { Mauvais remplissage des } \\
\text { pots et contamination par } \\
\text { des champignons ou des } \\
\text { levures, }\end{array}$ \\
\hline 4 & foncé & Cristallisé & verre & $\begin{array}{l}\text { Défaut de cristallisation } \\
\text { (Cristallisation aléatoire). }\end{array}$ & Choc thermique \\
\hline 5 & clair & $\begin{array}{l}\text { Liquide et } \\
\text { limpide }\end{array}$ & verre & Homogène & - \\
\hline 6 & foncé & Deux phases & verre & $\begin{array}{l}\text { Défaut de cristallisation } \\
\text { (séparation des phases) }\end{array}$ & Prise d'humidité \\
\hline 7 & foncé & Liquide & verre & $\begin{array}{l}\text { Défaut de cristallisation } \\
\text { (début de cristallisation } \\
\text { aléatoire) }\end{array}$ & Choc thermique \\
\hline 8 & verdâtre & Cristallisé & verre & $\begin{array}{l}\text { Cristallisation fine et } \\
\text { homogène }\end{array}$ & - \\
\hline 9 & foncé & $\begin{array}{l}\text { Liquide et } \\
\text { limpide }\end{array}$ & verre & Homogène & - \\
\hline 10 & foncé & Cristallisé & plastique & Homogène & - \\
\hline 11 & Clair & Cristallisé & verre & Contient de la cire d'abeille & - \\
\hline 12 & foncé & Cristallisé & $\begin{array}{l}\text { Bidon } \\
\text { métallique }\end{array}$ & $\begin{array}{l}\text { Défaut de cristallisation } \\
\text { (marbrure blanche) }\end{array}$ & $\begin{array}{l}\text { Choc thermique et manque } \\
\text { d'homogénéisation }\end{array}$ \\
\hline 13 & blanc & pâteux & verre & Homogène & - \\
\hline 14 & foncé & $\begin{array}{l}\text { Liquide et } \\
\text { limpide }\end{array}$ & verre & Homogène & - \\
\hline 15 & foncé & Liquide & $\begin{array}{l}\text { Bidon } \\
\text { métallique }\end{array}$ & Homogène & - \\
\hline 16 & Noir & Liquide & $\begin{array}{l}\text { Bidon } \\
\text { plastique }\end{array}$ & $\begin{array}{l}\text { Trop fluide avec odeur de } \\
\text { mélasse. }\end{array}$ & $\begin{array}{l}\text { Fermentation due à un } \\
\text { excès d'humidité et à une } \\
\text { mauvaise hygiène de } \\
\text { production. }\end{array}$ \\
\hline
\end{tabular}



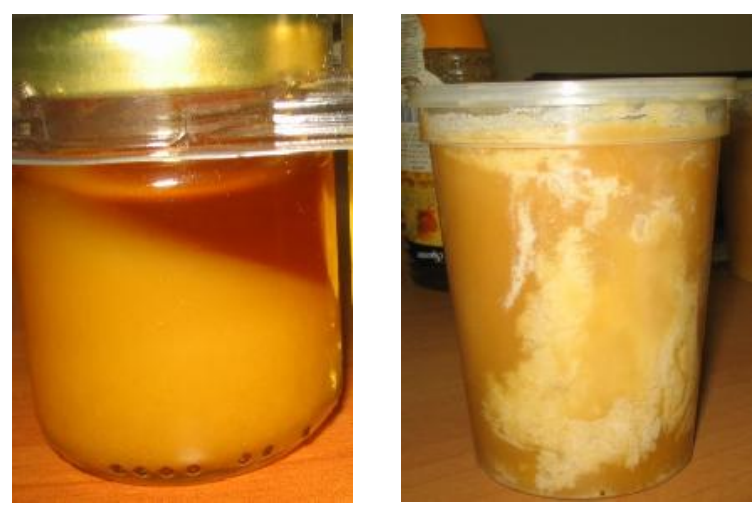

Fig.3. À gauche séparation des phases, À droite marbrures et cristallisation hétérogène

\subsection{Conformité de l'origine florale}

Dans la plupart des cas quant l'origine florale est mentionnée sur l'étiquette l'analyse pollinique à confirmé cette état de fait (tableau 3), sauf dans le cas où on ne trouve pas du tout de pollen, dénotant que ces miels ont été filtrés, comme le montre l'analyse des échantillons 17 et 30 . Le codex alimentarius [9] stipule clairement que les miels soumis à un filtrage minutieux pour améliorer la limpidité doivent porter obligatoirement la mention «filtré » sur l'étiquetage pour informer le consommateur, et c'est pour cela que les associations apicoles de l'UE conseillent dans leurs guides de bonnes pratiques l'utilisation des filtres dont la taille des pores est supérieure à $0,2 \mathrm{~mm}$.

Aussi, s'est posé le problème des pollens non identifiés pour beaucoup de ces échantillons (20, $21,22,23,24,25,26,40)$, car ils proviennent de pays dont on ne dispose pas d'atlas pollinique et dont la flore est très différente de celle de la méditerranée.

\subsection{Conformité de la qualité physico-chimique}

Le tableau 3 illustre la qualité des miels étudiés comparés aux exigences du codex Alimentarius, il s'avère que $40 \%$ des échantillons ont une humidité supérieure à $18 \%$, ce qui les rend susceptibles à la fermentation. Un seul échantillon a dépassé le seuil de $21 \%$ fixé comme valeur maximale, et qui présentait déjà des signes de fermentation avancée. L'humidité étant le critère le plus pertinent pour connaitre les aptitudes des miels au stockage [10]. 
Tableau 3: Résultats des analyses physico-chimiques et pollinique des miels étudiés

\begin{tabular}{|c|c|c|c|c|c|c|c|c|}
\hline Ech. & $\begin{array}{c}\text { Teneur } \\
\text { en eau } \\
(\%)\end{array}$ & $\mathrm{pH}$ & $\begin{array}{c}\mathrm{AL} \\
\text { (méq/kg) }\end{array}$ & $\begin{array}{c}\mathrm{CE} \\
(\mathrm{mS} / \mathrm{cm})\end{array}$ & $\begin{array}{c}\mathrm{HMF} \\
(\mathrm{mg} / \mathrm{kg})\end{array}$ & $\begin{array}{l}\text { proline } \\
(\mathrm{mg} / \mathrm{kg})\end{array}$ & $\begin{array}{c}\text { Indice de } \\
\text { couleur } \\
\text { (mm } \\
\text { Pfund) }\end{array}$ & $\begin{array}{l}\text { Origine } \\
\text { botanique }\end{array}$ \\
\hline 1 & 17.5 & 2,79 & 28 & 0,184 & 58,7 & 242 & 71 & Confirmé \\
\hline 2 & 16,4 & 2,86 & 30 & 0,179 & 70,4 & 343 & 83 & Confirmé \\
\hline 3 & 17,0 & 2,64 & 33 & 0,225 & 45,1 & 264 & 83 & Confirmé \\
\hline 4 & 18,1 & 2,33 & 28 & 0,239 & 121,0 & 300 & 92 & Confirmé \\
\hline 5 & 17,3 & 3,01 & 14 & 0,118 & 21,3 & 247 & 18 & Non confirmé \\
\hline 6 & 17,6 & 2,84 & 30 & 0,174 & 26,0 & 144 & 99 & Non identifié \\
\hline 7 & 17,6 & 2,44 & 36 & 0,218 & 72,2 & 365 & 77 & Confirmé \\
\hline 8 & 18,4 & 2,30 & 38 & 0,166 & 17,1 & 284 & 83 & Confirmé \\
\hline 9 & 17,4 & 3,06 & 13 & 0,084 & 46,1 & 73 & 80 & Confirmé \\
\hline 10 & 18,3 & 2,84 & 30 & 0,202 & 144,6 & 215 & 68 & Confirmé \\
\hline 11 & 18,0 & 2,59 & 20 & 0,143 & 72,6 & 77 & 71 & Confirmé \\
\hline 12 & 17,5 & 4,12 & 24 & 0,140 & 74,6 & 256 & 83 & Confirmé \\
\hline 13 & 17,0 & 4,43 & 13 & 0,079 & 23,5 & 102 & 11 & Confirmé \\
\hline 14 & 18,0 & 4,36 & 18 & 0,107 & 34,0 & 171 & 62 & Peu de pollen \\
\hline 15 & 16,1 & 4,82 & 14 & 0,100 & 102,1 & 33 & 83 & Confirmé \\
\hline 16 & 18,8 & 4,08 & 73 & 1,399 & 16,3 & 100 & 140 & Confirmé \\
\hline 17 & 15,0 & 4,00 & 49 & 0,367 & 13,5 & 33 & 62 & Pas de pollen \\
\hline 18 & 16,0 & 3,76 & 34 & 0,198 & 7,2 & 250 & 55 & Confirmé \\
\hline 19 & 20,5 & 4,77 & 75 & 0,334 & 34,6 & 200 & 119 & Confirmé \\
\hline 20 & 21 & 5,13 & 55 & 0,534 & 67,2 & 300 & 119 & Non identifié \\
\hline 21 & 21 & 5,14 & 56 & 0,538 & 80,4 & 200 & 119 & Non identifié \\
\hline 22 & 21 & 5,22 & 58 & 0,568 & 59,7 & 150 & 119 & Non identifié \\
\hline 23 & 21 & 5,12 & 75 & 0,570 & 96,4 & 300 & 119 & Non identifié \\
\hline 24 & 18,5 & 5,40 & 44 & 0,625 & 20,2 & 250 & 110 & Non identifié \\
\hline 25 & 19,5 & 4,86 & 33 & 0,177 & 86,8 & 100 & 92 & Non confirmé \\
\hline 26 & 18 & 5,18 & 38 & 0,204 & 61,4 & 110 & 83 & Non identifié \\
\hline 27 & 18,5 & 5,06 & 43 & 0,145 & 106,3 & 250 & 92 & Non identifié \\
\hline 28 & 18,0 & 4,90 & 85 & 0,381 & 41 & 350 & 62 & Confirmé \\
\hline 29 & 22,0 & 5,12 & 57 & 0,647 & 101 & 200 & 110 & Non identifié \\
\hline 30 & 19,0 & 4,73 & 42 & 0,102 & 50,4 & 100 & 62 & Pas de pollen \\
\hline 31 & 17 & 4,42 & 33 & 0,513 & 51,9 & 284 & 119 & Confirmé \\
\hline 32 & 18,5 & 3,83 & 24 & 0,264 & 35,8 & 300 & 62 & non confirmé \\
\hline 33 & 19,5 & 3,93 & 15 & 0,158 & 67,8 & 207 & 83 & Confirmé \\
\hline 34 & 17,8 & 4,78 & 13 & 0,187 & 16,5 & 220 & 83 & Confirmé \\
\hline 35 & 18,2 & 4,00 & 19 & 0,244 & 65,0 & 240 & 83 & Confirmé \\
\hline 36 & 15,8 & 4,96 & 41 & 0,760 & 0,4 & 200 & 119 & non confirmé \\
\hline 37 & 18,4 & 4,85 & 15 & 0,123 & 9,6 & 300 & 41 & Confirmé \\
\hline 38 & 17,2 & 3,85 & 12,5 & 0,100 & 22,5 & 120 & 71 & Non confirmé \\
\hline 39 & 18,5 & 4,20 & 7 & 0,07 & 51,2 & 180 & 11 & Confirmé \\
\hline 40 & 15,5 & 6,81 & 9 & 0,77 & 157,2 & 120 & 110 & Non identifié \\
\hline Max & 22 & 6,81 & 85 & 1,399 & 157,2 & 365 & 140 & \\
\hline Min & 15 & 2,3 & 7 & $\mathbf{0 , 0 7 0}$ & 0,4 & 33 & 11 & \\
\hline $\mathrm{M} \pm \mathrm{SD}$ & $18,2 \pm 1,6$ & $4,1 \pm 1,1$ & $34,4 \pm 20,1$ & $0,308 \pm 0,267$ & $56,2 \pm 37,6$ & $207,7 \pm 102,8$ & $82,6 \pm 30,3$ & \\
\hline Normes & $\underline{21}$ & $\begin{array}{l}3,5- \\
5,5\end{array}$ & $\leq 40$ & & $<40$ & $>183$ & & \\
\hline
\end{tabular}

M : moyenne ; SD : ecart type ; Ech. : échantillon 
Le $\mathrm{pH}$ et la $\mathrm{CE}$ sont des paramètres qui renseignent sur l'origine florale des miels, ceux issus de nectar étant, généralement, plus acides et moins riches en minéraux comparés aux miellats. Alors que le $\mathrm{pH}$ change avec le temps et deviens plus bas avec le vieillissement, cas de plus de $27 \%$ des échantillons étudiés dont le $\mathrm{pH}$ est inférieur à 3,5; la conductivité électrique reste relativement stable et nous a servi à confirmer les miellats $\left(\mathrm{CE}>0,800 \mathrm{mS} . \mathrm{cm}^{-1}\right)$. Dans deux cas, on se rend compte que la mention «miel de foret » ne renvoi pas, forcément, à des miellats mais à des miels de nectar (ech. 38) ou à des mélanges nectar/miellat (ech. 36), ce qui peut être une source de confusion pour le consommateur.

La proline est utilisée pour détecter les fraudes par ajout de sucres invertis aux miels [11] ; elle varie naturellement dans un large intervalle mais des taux plus bas que $183 \mathrm{mg} / \mathrm{kg}$ indiquent une anomalie. Les échantillons 9, 11, 13 et 15 présentent des taux de proline extrêmement faibles qui sont respectivement $73,77,102$ et $33 \mathrm{mg} / \mathrm{kg}$, et des CE très basses $(0,084 ; 0,143 ; 0,079$ et $\left.0,100 \mathrm{mS} . \mathrm{cm}^{-1}\right)$, indiquant un mélange avec des sucres invertis ou du sirop de saccharose qui ne contiennent ni proline, ni minéraux [12] ; de ce fait, ils ne peuvent plus porter la dénomination légale « miel ».

En dernier, le taux d'HMF, qui est le critère le plus pertinent quant à la fraicheur des miels et à leur historique thermique [13] [14], s'est avéré trop élevé avec une moyenne, pour tous les échantillons, de 56,2 $\pm 37,6 \mathrm{mg} / \mathrm{kg}$, sur une étendue allant de 0,4 à 157,2 mg/kg. Nous savons que le miel atteint sa fin de vie vers $40 \mathrm{mg} / \mathrm{kg}$ et ne peut plus porter la dénomination « miel », mais il peut être réorienté vers l'industrie alimentaire en tant que «miel industriel».

Pour donner une image la plus juste sur ces échantillons, il convient de signaler que $30 \%$ des échantillons ont un taux dans la norme $(<40 \mathrm{mg} / \mathrm{kg})$ parmi lesquels seulement un tiers peuvent être classés dans les miels de qualité (HMF< 15mg/kg) [15].

Par opposition, 70\% de ces miels ont largement dépassé leur DLUO avec un taux moyen de $78,0 \pm 30,3 \mathrm{mg} / \mathrm{kg}$ d'HMF, dénotant d'un vieillissement avancé (circuit économique long), de traitements post-récolte inappropriés (refonte, pasteurisation) ou de mauvaises conditions de stockages et de transport (expositions prolongée au soleil, stockage dans les conteneurs métalliques...etc.), sachant que ces denrées importées peuvent mettre des mois pour arriver sur nos étalages.

\section{CONCLUSION}

Au terme de ce travail, on peut faire ressortir les faits les plus pertinents quant à la qualité des miels importés mis à la disposition du consommateur Algériens. Alors que plus de $60 \%$ de ces 
miels répondent aux attentes du consommateur du point de vu présentation, emballage, couleur et prix accessible à toutes les bourses, il n'en est pas autant pour leur qualité car :

- $60 \%$ ne sont pas conforme du point de vu étiquetage et peuvent rendre difficile l'accès à l'information pour le consommateur, et peuvent créer chez lui une confusion quant à l'origine florale ou territoriale.

- Plus de $40 \%$ présentent des défauts organoleptiques et de cristallisation.

- Pour près de $45 \%$ l'origine botanique n'a pas pu être confirmé, soit à cause de l'absence de pollen dans ces miels, ou à cause de l'impossibilité d'identifier les pollens présents par manque de références polliniques des pays en question.

- $\quad 10 \%$ sont mélangés avec des sucres invertis, ce qui relève de la fraude.

- Plus de 70\% ont dépassés leur DLUO, car ils présentent des taux supérieurs à 40mg/kg d'HMF et sont devenus trop acides, ils ne peuvent plus être vendus sous la dénomination « miel».

Ceci ne nous permet pas de généraliser notre jugement sur les miels d'importation, mais il nous montre, clairement, que ces miels bien présentés, bien emballés et bon marché, peuvent être d'une qualité très médiocre et parfois impropres à la consommation. Ça nous permet aussi de tirer une sonnette d'alarme quant à la nécessité du contrôle rigoureux des miels d'importation car il ressort, surtout, de la protection de nos consommateurs et de la viabilité de notre apiculture.

\section{REMERCIEMENTS}

Nous tenons à remercier pour leur aide précieuse, Mme Boucheffa Asma ITELV, les étudiants ayant participé à ce travail, le personnel des laboratoires LRTA et du département de technologie alimentaire de la FSI, Université M'hamed Bougara Boumerdes.

\section{RÉFERENCES BIBLIOGRAPHIQUES}

[1] Crane E., Bees and beekeeping. Heinemann newnes, Oxford, Heinemann Professional publishing Ltd., 1990, 614p.

[2] Gonnet M., Le miel : composition, propriétés, conservation. Edition OPIDA, 2éme éd., 1982, 32p.

[3] DSV/DSCSHA, Document du ministère de l'agriculture et du développement rural sur la filière apicole en Algérie. 2012.

[4] Bogdanov S., Martin P. and Lüllmann C., Apidologie.1997, 1S-59S.

[5] Von Der Ohe W., Persano Odo L., Piana M.L., Morlt M. et Martin P. Apidologie, 2004, 35, S18-S25. 
[6] Riccardelli d'albore G., text book of mediteranean melissopalynology, università degli Studi di Perugia. 1997, 466p.

[7] JORA, $N^{\circ} 58$ du 9 novembre 2013, décret $N^{\circ}$ 13/378, article 7 sur l'étiquetage des produits alimentaires et le droit du consommateur à l'information.

[8] Haderbache L., Mohammedi A., Enquête sur le profile du consommateur algérien In Thèse sur la qualité des miels algériens, DTA, FSI, UMBB. 2014.

[9] Codex Alimentarius, standards for honey, FAO-WHO, Rome. 1993.

[10] Bogdanov S., Ruoff K., Persano Oddo L., Apidologie. 2004, 35, S4-S17.

[11] Von Der Ohe W., Dustmann J. H.,Von Der Ohe K., Dtsch. Lebensm. Rundsch. 1991, 87, 383-386.

[12] Anklam E., Food Chemistry. 1998, 63, 549-562.

[13] Jeanne f., Bul. Techn. Apic. 1993, 18 (4), 77, 221-224.

[14] Gonnet M., Revue française d'apiculture. 1993, 30 , 269-271.

[15] Lachman J., Kolihovà D., Kosăta J., Titěra D., Kult k, Food chemistry. 2007, 101, 973 979.

How to cite this article

Haderbache L, Mohammedi A. Quality of imported honeys marketed in Algeria. J Fundam Appl Sci. 2015, 7(1), 139-149. 